\title{
Optimal consumption and portfolio selection with lower and upper bounds on consumption
}

\author{
Kum-Hwan Roh and Yong Hyun Shin ${ }^{2 *}$ (D)
}

\section{"Correspondence:}

yhshin@sookmyung.ac.kr

${ }^{2}$ Department of Mathematics \&

Research Institute of Natural

Sciences, Sookmyung Women's

University, Seoul, 04310, Republic of

Korea

Full list of author information is

available at the end of the article

\begin{abstract}
We investigate the optimal consumption and investment problem with lower and upper bounds on consumption constraints. We derive closed-form solutions by means of the dynamic programming approach. We also evaluate the effects of the optimal consumption and portfolio on consumption constraints and present some numerical/economic implications. In particular, we see that the upper bound on consumption acts as a bliss level in a quadratic utility model.
\end{abstract}

Keywords: Consumption constraints; Consumption/Investment problem; CRRA utility; Dynamic programming approach

\section{Introduction}

After Merton's pioneer research $[9,10]$ on continuous-time portfolio selection, there have been many studies conducted on the optimal consumption and portfolio selection problem with realistic economic constraints such as borrowing constraints, subsistence consumption constraints, portfolio constraints, etc. In this paper we focus on consumption constraints in particular.

When observing the consumption behavior of the economic agent, it is natural to consider the subsistence consumption requirement. A subsistence consumption constraint means that the agent should consume a positive minimum consumption requirement which the agent can live with. Some authors have previously investigated the portfolio selection problem with a subsistence consumption constraint (Gong and Li [2], Lakner and Nygren [7], Yuan and Hu [15], Shin et al. [13], etc).

When the optimal consumption and investment problem with quadratic utility is considered, a constant bliss level of consumption depending upon the coefficient of the quadratic utility function can be presented. In this level, the agent has zero investment, but consumes a constant bliss level (Koo et al. [6], Shin et al. [12]). This is the reason for our interest in an upper bound on consumption. Since we have already considered the agent who has a constraint which is acting like a bliss level in quadratic utility [12], we investigate the aspects of the optimal consumption and investment policy of agent when considering an upper bound on consumption. In the real financial market, there are cer-

(c) The Author(s) 2020. This article is licensed under a Creative Commons Attribution 4.0 International License, which permits use, sharing, adaptation, distribution and reproduction in any medium or format, as long as you give appropriate credit to the original author(s) and the source, provide a link to the Creative Commons licence, and indicate if changes were made. The images or other third party material in this article are included in the article's Creative Commons licence, unless indicated otherwise in a credit line to the material. If material is not included in the article's Creative Commons licence and your intended use is not permitted by statutory regulation or exceeds the permitted use, you will need to obtain permission directly from the copyright holder. To view a copy of this licence, visit http://creativecommons.org/licenses/by/4.0/. 
tain goods which cannot be infinitely consumed, such as foods, houses, and cars. Thus, the upper bound on consumption has an economic meaning when the agent consumes necessary goods. Jian et al. [4] and $\mathrm{Xu}$ and $\mathrm{Yi}$ [14] considered the optimal consumption and portfolio selection problem with an upper bound consumption constraint, which is a constant/a linear function of wealth, respectively. Unlike the bliss level on consumption, this constraint implies a free boundary problem. In this paper, we take the portfolio selection problem into account with the constant upper bound on consumption. In this paper we want to combine two previous issues which are a subsistence consumption constraint and a bliss level of consumption in the continuous-time portfolio selection problem, that is, we consider both the upper and lower bounds on consumption simultaneously to formulate these two issues.

Ma et al. [8] also considered the optimal consumption and investment problem with lower and upper bounds on consumption. Mathematically, Ma et al. [8] solved the optimization problem using a dual transformation, while we use the dynamic programming method. Furthermore, we observe the numerical/economic implications of the optimal consumption and portfolio with CRRA utility, and we can observe behaviors of the optimal consumption and portfolio, which are similar to the results with quadratic utility $[6,12]$.

Bensoussan et al. [1] and Jang et al. [3] solved the optimal consumption and portfolio selection problem in an incomplete market. Bensoussan et al. [1] considered involuntary unemployment risk which was not able to be hedged in a market. In the paper of Jang et al. [3], default risk of the provider of the annuity made a market to be incomplete. Using those approaches, it is possible to extend our results to the labor income problem with voluntary/involuntary retirement jointly.

The rest of this paper is composed as follows. We represent the financial market in Sect. 2. We derive our optimization problem and solve this problem by means of the dynamic programming approach proposed by Karatzas et al. [5] in Sect. 3. Then, Sect. 4 gives some numerical implications, and Sect. 5 brings to a conclusion.

\section{The economy}

Assume that there are a riskless asset $P$ and a risky asset $S$ in the financial market. A riskless asset $P_{t}$ at time $t$ follows the ordinary differential equation (ODE)

$$
d P_{t}=r P_{t} d t
$$

where $r>0$ is an interest rate, and a risky asset $S_{t}$ at time $t$ evolves according to

$$
d S_{t}=b S_{t} d t+\sigma S_{t} d Z_{t}
$$

where $b>r$ is a mean rate of return and $\sigma$ is a volatility of the risky asset. All of the parameters, $r, b$, and $\sigma$, are assumed to be constants. $Z_{t}$ is the standard Brownian motion on a probability space $(\Omega, \mathcal{F}, \mathbb{P})$ and $\left\{\mathcal{F}_{t}\right\}_{t \geq 0}$ is the $\mathbb{P}$-augmentation of the filtration generated by the standard Brownian motion $\left\{Z_{t}\right\}_{t \geq 0}$.

Let $\pi_{t}$ be the amounts of money (dollar) invested in the risky asset $S_{t}$ at time $t$, and let $c_{t} \geq 0$ be the consumption rate process at time $t$. We assume that the portfolio process $\pi_{t}$ 
at time $t$ is an $\mathcal{F}_{t}$-measurable process, that the consumption process $c_{t}$ at time $t$ is an $\mathcal{F}_{t^{-}}$ measurable nonnegative process, and that these processes satisfy the following technical conditions:

$$
\int_{0}^{t} \pi_{s}^{2} d s<\infty, \quad \int_{0}^{t} c_{s} d s<\infty \quad \text { for all } t \geq 0 \text { a.s. }
$$

Then, the wealth process of the agent $X_{t}$ at time $t$ evolves according to

$$
d X_{t}=\left[r X_{t}+\pi_{t}(b-r)-c_{t}\right] d t+\sigma \pi_{t} d Z_{t}
$$

with an initial endowment $X_{0}=x>0$.

\section{The optimization problem}

The goal of the agent is to maximize her/his lifetime discounted expected utility

$$
J(x ; c, \pi):=\mathbb{E}\left[\int_{0}^{\infty} e^{-\beta t} \frac{c_{t}^{1-\gamma}}{1-\gamma} d t\right]
$$

subject to the budget constraint (2.1) and the consumption constraint that restrains the consumption behavior such that

$$
\underline{B} \leq c_{t} \leq \bar{B} \text { for all } t \geq 0 \text {, }
$$

where $\underline{B}>0$ and $\bar{B}>\underline{B}$ are positive constants; that is, the upper bound of consumption as well as the lower bound of consumption. Here, $\beta>0$ is a subjective discount factor and $\gamma>0(\gamma \neq 1)$ is an agent's coefficient of relative risk aversion. The pair $(c, \pi)$ of the optimal consumption and portfolio is called admissible at initial wealth $x>0$ if $X_{t}>0$ for all $t \geq 0$ and if it satisfies constraint (3.1).

Thus, the value function of our optimization problem can be given by

$$
V(x):=\sup _{(c, \pi) \in \mathcal{A}(x)} J(x ; c, \pi)
$$

where $\mathcal{A}(x)$ is the class of all admissible pairs $(c, \pi)$ at $x>0$ such that

$$
\mathbb{E}\left[\int_{0}^{\infty} e^{-\beta t}\left(\frac{c_{t}^{1-\gamma}}{1-\gamma}\right)^{-} d t\right]<\infty,
$$

where $z^{-}:=\max (-z, 0)$.

Assumption 1 (Gong and Li [2]) There exists a positive lower bound of initial wealth $x>0$ such that

$$
x>\frac{B}{r} .
$$

From the value function (3.2), constraints (2.1) and (3.1), we derive the Bellman equation as follows:

$$
\max _{(\underline{B} \leq c \leq \bar{B}, \pi)}\left[\{r x+\pi(b-r)-c\} V^{\prime}(x)+\frac{1}{2} \sigma^{2} \pi^{2} V^{\prime \prime}(x)-\beta V(x)+\frac{c^{1-\gamma}}{1-\gamma}\right]=0 .
$$


The first order conditions (FOCs) imply

$$
c^{*}= \begin{cases}\underline{B} & \text { for } \underline{B} / r<x<\underline{z} \\ \left(V^{\prime}(x)\right)^{-\frac{1}{\gamma}} & \text { for } \underline{z} \leq x<\bar{z} \\ \bar{B} & \text { for } x \geq \bar{z}\end{cases}
$$

and

$$
\pi^{*}=-\frac{b-r}{\sigma^{2}} \frac{V^{\prime}(x)}{V^{\prime \prime}(x)}
$$

where $\underline{z}>0$ is the wealth level corresponding to the lower consumption level $\underline{B}, \bar{z}>0$ is the wealth level corresponding to the upper consumption level $\bar{B}$, and $\underline{z}<\bar{z}$ ( $\underline{z}$ and $\bar{z}$ will be determined later in Theorem 1). Inserting FOCs (3.4) and (3.5) into equation (3.3) yields

$$
\begin{aligned}
& (r x-\underline{B}) V^{\prime}(x)-\frac{1}{2} \theta^{2} \frac{\left(V^{\prime}(x)\right)^{2}}{V^{\prime \prime}(x)}-\beta V(x)+\frac{\underline{B}^{1-\gamma}}{1-\gamma}=0 \quad \text { for } \underline{B} / r<x<\underline{z}, \\
& r x V^{\prime}(x)-\frac{1}{2} \theta^{2} \frac{\left(V^{\prime}(x)\right)^{2}}{V^{\prime \prime}(x)}-\beta V(x)+\frac{\gamma}{1-\gamma} V^{\prime}(x)^{-\frac{1-\gamma}{\gamma}}=0 \quad \text { for } \underline{z} \leq x<\bar{z},
\end{aligned}
$$

and

$$
(r x-\bar{B}) V^{\prime}(x)-\frac{1}{2} \theta^{2} \frac{\left(V^{\prime}(x)\right)^{2}}{V^{\prime \prime}(x)}-\beta V(x)+\frac{\bar{B}^{1-\gamma}}{1-\gamma}=0 \quad \text { for } x \geq \bar{z},
$$

where $\theta:=(b-r) / \sigma$ is Sharpe ratio (or market price of risk). Moreover, we define the constant $K>0$ as follows:

$$
K:=r+\frac{\beta-r}{\gamma}+\frac{\gamma-1}{2 \gamma^{2}} \theta^{2}>0
$$

Remark 1 For later use, we define two quadratic functions as follows:

$$
f(m):=r m^{2}-\left(\beta+r+\frac{1}{2} \theta^{2}\right) m+\beta
$$

and

$$
g(n):=\frac{1}{2} \theta^{2} n^{2}+\left(\beta-r+\frac{1}{2} \theta^{2}\right) n-r .
$$

The quadratic equation $f(m)=0$ has two roots $m_{1}$ and $m_{2}\left(m_{1}>1>m_{2}>0\right)$ and the quadratic equation $g(n)=0$ has two roots $n_{1}>0$ and $n_{2}<-1$. In addition, we have the following relationships between the roots of $f(m)=0$ and the roots of $g(n)=0$ :

$$
n_{1}=\frac{1}{m_{1}-1}, \quad n_{2}=\frac{1}{m_{2}-1},
$$

and

$$
\frac{n_{1}}{n_{1}+1}=\frac{r-\frac{1}{2} \theta^{2} n_{1}}{\beta}, \quad \frac{n_{2}}{n_{2}+1}=\frac{r-\frac{1}{2} \theta^{2} n_{2}}{\beta} .
$$


Theorem 1 The value function $V(x)$ of the optimization problem (3.2) is given by

$$
V(x)= \begin{cases}C_{2}\left(x-\frac{B}{r}\right)^{m_{2}}+\frac{\underline{B}^{1-\gamma}}{\beta(1-\gamma)} & \text { for } \underline{B} / r<x<\underline{z} \\ \frac{n_{1}}{n_{1}+1} D_{1} \xi^{-\gamma\left(n_{1}+1\right)}+\frac{n_{2}}{n_{2}+1} D_{2} \xi^{-\gamma\left(n_{2}+1\right)}+\frac{1}{(1-\gamma) K} \xi^{1-\gamma} & \text { for } \underline{z} \leq x \leq \bar{z}, \\ E_{1}\left(\frac{\bar{B}}{r}-x\right)^{m_{1}}+\frac{\bar{B}^{1-\gamma}}{\beta(1-\gamma)} & \text { for } \bar{z}<x<\bar{B} / r \\ \frac{\bar{B}^{1-\gamma}}{\beta(1-\gamma)} & \text { for } x \geq \bar{B} / r\end{cases}
$$

where

$$
\begin{aligned}
& D_{1}=\frac{\frac{1}{K}+\gamma n_{2}\left(\frac{1}{K}-\frac{1}{r}\right)}{\gamma\left(n_{1}-n_{2}\right)} \underline{B}^{1+\gamma n_{1},}, \\
& D_{2}=\frac{\frac{1}{K}+\gamma n_{1}\left(\frac{1}{K}-\frac{1}{r}\right)}{\gamma\left(n_{2}-n_{1}\right)} \bar{B}^{1+\gamma n_{2}}, \\
& \underline{z}=D_{1} \underline{B}^{-\gamma n_{1}}+D_{2} \underline{B}^{-\gamma n_{2}}+\frac{\underline{B}}{K}, \\
& \bar{z}=D_{1} \bar{B}^{-\gamma n_{1}}+D_{2} \bar{B}^{-\gamma n_{2}}+\frac{\bar{B}}{K}, \\
& C_{2}=\frac{1}{m_{2}}\left(\underline{z}-\frac{\underline{B}}{r}\right)^{1-m_{2}} \underline{B}^{-\gamma}>0,
\end{aligned}
$$

and

$$
E_{1}=-\frac{1}{m_{1}}\left(\frac{\bar{B}}{r}-\bar{z}\right)^{1-m_{1}} \bar{B}^{-\gamma}<0 .
$$

For $\underline{z} \leq x<\bar{z}, \xi$ is determined by the algebraic equation as follows:

$$
x=D_{1} \xi^{-\gamma n_{1}}+D_{2} \xi^{-\gamma n_{2}}+\frac{1}{K} \xi
$$

Proof For $\underline{B} / r<x<\underline{z}$, if we conjecture the form of the solution to equation (3.6) as follows:

$$
V(x)=C_{1}\left(x-\frac{\underline{B}}{r}\right)^{m_{1}}+C_{2}\left(x-\frac{\underline{B}}{r}\right)^{m_{2}}+\frac{\underline{B}^{1-\gamma}}{\beta(1-\gamma)},
$$

then it solves equation (3.6) when $C_{1}=0$ and $C_{2}>0$. Thus, $V(x)$ is given by

$$
V(x)=C_{2}\left(x-\frac{B}{r}\right)^{m_{2}}+\frac{\underline{B}^{1-\gamma}}{\beta(1-\gamma)},
$$

where $m_{1}$ and $m_{2}$ are the two roots of the quadratic equation $f(m)=0$ in (3.10), and $C_{2}$ is a constant which will be determined later.

In the case of $x \geq \bar{z}, x-\bar{B} / r$ should be negative in the interval $(\bar{z}, \bar{B} / r)$. Hence we suppose that the form of the solution to equation (3.8) is given as follows:

$$
V(x)=E_{1}\left(\frac{\bar{B}}{r}-x\right)^{m_{1}}+E_{2}\left(\frac{\bar{B}}{r}-x\right)^{m_{2}}+\frac{\bar{B}^{1-\gamma}}{\beta(1-\gamma)} .
$$


By the property $\lim _{x \rightarrow \bar{B} / r} V^{\prime}(x)<\infty, E_{2}=0$. Hence, we obtain $V(x)$ as follows:

$$
V(x)=E_{1}\left(\frac{\bar{B}}{r}-x\right)^{m_{1}}+\frac{\bar{B}^{1-\gamma}}{\beta(1-\gamma)} .
$$

For $x>\bar{B} / r$, similar to the case of $\underline{B} / r<x<\underline{z}$, if we suppose that the form of the solution to equation (3.8) is given as follows:

$$
V(x)=F_{1}\left(x-\frac{\bar{B}}{r}\right)^{m_{1}}+F_{2}\left(x-\frac{\bar{B}}{r}\right)^{m_{2}}+\frac{\bar{B}^{1-\gamma}}{\beta(1-\gamma)},
$$

then it solves equation (3.8) when $F_{1}=0$ and $F_{2}=0$ because of $\lim _{x \rightarrow \infty} V(x)<\infty$. Thus, $V(x)$ is given by

$$
V(x)=\frac{\bar{B}^{1-\gamma}}{\beta(1-\gamma)} .
$$

We now find the solution to equation (3.7) for $\underline{z} \leq x<\bar{z}$. First, we assume that the optimal consumption is a function of optimal wealth, that is, $c=C(x)$ and $X(\cdot)=C^{-1}(\cdot)$. From FOC (3.4), we have

$$
V^{\prime}(x)=C(x)^{-\gamma}, \quad V^{\prime \prime}(x)=-\gamma \frac{C(x)^{-\gamma-1}}{X^{\prime}(c)} .
$$

Substituting equations (3.18) into equation (3.7) implies

$$
\beta V(X(c))=r c^{-\gamma} X(c)+\frac{1}{2 \gamma} \theta^{2} c^{1-\gamma} X^{\prime}(c)+\frac{\gamma}{1-\gamma} c^{1-\gamma}
$$

By differentiating equation (3.19) with respect to $c$, we obtain the following second order ODE:

$$
\frac{1}{2 \gamma} \theta^{2} c^{2} X^{\prime \prime}(c)-\left(\beta-r-\frac{1-\gamma}{2 \gamma} \theta^{2}\right) c X^{\prime}(c)-\gamma r X(c)+\gamma c=0 .
$$

Then, we can obtain the solution to equation (3.20) as follows:

$$
X(c)=D_{1} c^{-\gamma n_{1}}+D_{2} c^{-\gamma n_{2}}+\frac{1}{K} c,
$$

where $n_{1}$ and $n_{2}$ are the two roots of the quadratic equation $g(n)=0$ in (3.11), and $D_{1}$ and $D_{2}$ are constants which will be determined later. Inserting equation (3.21) into equation (3.19) implies

$$
\begin{aligned}
V(x) & =\frac{r-\frac{1}{2} \theta^{2} n_{1}}{\beta} D_{1} \xi^{-\gamma\left(n_{1}+1\right)}+\frac{r-\frac{1}{2} \theta^{2} n_{2}}{\beta} D_{2} \xi^{-\gamma\left(n_{2}+1\right)}+\frac{1}{(1-\gamma) K} \xi^{1-\gamma} \\
& =\frac{n_{1}}{n_{1}+1} D_{1} \xi^{-\gamma\left(n_{1}+1\right)}+\frac{n_{2}}{n_{2}+1} D_{2} \xi^{-\gamma\left(n_{2}+1\right)}+\frac{1}{(1-\gamma) K} \xi^{1-\gamma},
\end{aligned}
$$


where the second equality is obtained from the relationships (3.13) and $\xi$ is determined from the algebraic equation

$$
x=D_{1} \xi^{-\gamma n_{1}}+D_{2} \xi^{-\gamma n_{2}}+\frac{1}{K} \xi .
$$

From (3.21), we obtain the wealth levels $\underline{z}$ and $\bar{z}$ :

$$
\underline{z}=X(\underline{B})=D_{1} \underline{B}^{-\gamma n_{1}}+D_{2} \underline{B}^{-\gamma n_{2}}+\frac{\underline{B}}{K}, \quad \bar{z}=X(\bar{B})=D_{1} \bar{B}^{-\gamma n_{1}}+D_{2} \bar{B}^{-\gamma n_{2}}+\frac{\bar{B}}{K} .
$$

Based on equations (3.17) and (3.18) as well as the $C^{1}$ - and $C^{2}$-conditions of $V(x)$ at $\underline{z}$, we have the following equations:

$$
V^{\prime}(\underline{z})=C_{2} m_{2}\left(\underline{z}-\frac{\underline{B}}{r}\right)^{m_{2}-1}=\underline{B}^{-\gamma}
$$

and

$$
V^{\prime \prime}(\underline{z})=C_{2} m_{2}\left(m_{2}-1\right)\left(\underline{z}-\frac{\underline{B}}{r}\right)^{m_{2}-2}=-\gamma \frac{\underline{B}^{-\gamma-1}}{X^{\prime}(\underline{B})} .
$$

Two equations (3.23) and (3.24) imply

$$
C_{2}=\frac{1}{m_{2}}\left(\underline{z}-\frac{\underline{B}}{r}\right)^{1-m_{2}} \underline{B}^{-\gamma}>0
$$

and

$$
\underline{B} \cdot X^{\prime}(\underline{B})=-\gamma n_{1} D_{1} \underline{B}^{-\gamma n_{1}}-\gamma n_{2} D_{2} \underline{B}^{-\gamma n_{2}}+\frac{1}{K} \underline{B}=-\frac{\gamma}{m_{2}-1}\left(\underline{z}-\frac{\underline{B}}{r}\right) .
$$

Substituting $\underline{z}$ in (3.22) into equation (3.25) implies

$$
D_{1}=\frac{\frac{1}{K}+\gamma n_{2}\left(\frac{1}{K}-\frac{1}{r}\right)}{\gamma\left(n_{1}-n_{2}\right)} \underline{B}^{1+\gamma n_{1}},
$$

since $n_{2}=1 /\left(m_{2}-1\right)$ in (3.12). Similarly, we can also obtain $E_{1}$ and $D_{2}$ as follows:

$$
\begin{aligned}
& E_{1}=-\frac{1}{m_{1}}\left(\frac{\bar{B}}{r}-\bar{z}\right)^{1-m_{1}} \bar{B}^{-\gamma}<0, \\
& D_{2}=\frac{\frac{1}{K}+\gamma n_{1}\left(\frac{1}{K}-\frac{1}{r}\right)}{\gamma\left(n_{2}-n_{1}\right)} \bar{B}^{1+\gamma n_{2}} .
\end{aligned}
$$

According to Remark 3.3 in Shim and Shin [11], we obtain the following lemma.

Lemma $1 D_{1}>0$ in (3.14).

Proof Since $n_{1}-n_{2}>0$, it is sufficient to show that

$$
\frac{1}{K}+\gamma n_{2}\left(\frac{1}{K}-\frac{1}{r}\right)>0 .
$$


For $n_{2}<x<n_{1}$, we define a function

$$
h(x):=-\frac{g(x)}{x-n_{2}}=-\frac{1}{2} \theta^{2}\left(x-n_{1}\right)>0,
$$

with the quadratic function $g(\cdot)$ in (3.11). Then, $h(x)$ is a linearly decreasing function for $n_{2}<x<n_{1}$. Since $g(-1 / \gamma)=-K<0$ and $n_{2}<-1 / \gamma<0<n_{1}$, we can see that $h(-1 / \gamma)>$ $h(0)>0$ and

$$
-\frac{K}{\frac{1}{\gamma}+n_{2}}>-\frac{r}{n_{2}}>0
$$

that is,

$$
0<\frac{-\frac{1}{\gamma}-n_{2}}{K}<-\frac{n_{2}}{r} \text {. }
$$

Thus, inequality (3.29) implies inequality (3.28).

Lemma $2 D_{2}<0$ in (3.15).

Proof It is similar to the proof of Lemma 1.

\section{Lemma 3}

$$
\bar{z}<\frac{\bar{B}}{r}
$$

Proof From (3.16), (3.14), and (3.15), we see that

$$
\begin{aligned}
\bar{z} & =\frac{\frac{1}{K}+\gamma n_{2}\left(\frac{1}{K}-\frac{1}{r}\right)}{\gamma\left(n_{1}-n_{2}\right)} \underline{B}^{1+\gamma n_{1}} \bar{B}^{-\gamma n_{1}}+\frac{\frac{1}{K}+\gamma n_{1}\left(\frac{1}{K}-\frac{1}{r}\right)}{\gamma\left(n_{2}-n_{1}\right)} \bar{B}^{1+\gamma n_{2}} \bar{B}^{-\gamma n_{2}}+\frac{\bar{B}}{K} \\
& =\left[\frac{\frac{1}{K}+\gamma n_{2}\left(\frac{1}{K}-\frac{1}{r}\right)}{\gamma\left(n_{1}-n_{2}\right)}\left(\frac{B}{\bar{B}}\right)^{1+\gamma n_{1}}+\frac{\frac{1}{K}+\gamma n_{1}\left(\frac{1}{K}-\frac{1}{r}\right)}{\gamma\left(n_{2}-n_{1}\right)}+\frac{1}{K}\right] \bar{B} \\
& <\left[\frac{\frac{1}{K}+\gamma n_{2}\left(\frac{1}{K}-\frac{1}{r}\right)}{\gamma\left(n_{1}-n_{2}\right)}+\frac{\frac{1}{K}+\gamma n_{1}\left(\frac{1}{K}-\frac{1}{r}\right)}{\gamma\left(n_{2}-n_{1}\right)}+\frac{1}{K}\right] \bar{B} \\
& =\frac{\bar{B}}{r}
\end{aligned}
$$

where the inequality is obtained from (3.28) and $\underline{B} / \bar{B}<1$.

Lemma $4 X^{\prime}(c)>0$ for $\underline{B}<c<\bar{B}$.

Proof We derive $X^{\prime \prime}(\cdot)$ from $X(\cdot)$ in (3.21) as follows:

$$
X^{\prime \prime}(c)=c^{-2}\left[\gamma n_{1}\left(\gamma n_{1}+1\right) D_{1} c^{-\gamma n_{1}}+\gamma n_{2}\left(\gamma n_{2}+1\right) D_{2} c^{-\gamma n_{2}}\right]=: c^{-2} F(c) \text {. }
$$

Since $D_{2}<0, F(c)=0$ has a unique solution $c^{\star}>0$ such that

$$
c^{\star}=\left[-\frac{n_{2}\left(\gamma n_{2}+1\right) D_{2}}{n_{1}\left(\gamma n_{1}+1\right) D_{1}}\right]^{-\frac{1}{\gamma\left(n_{1}-n_{2}\right)}} .
$$


Since $\lim _{c \rightarrow 0+} F(c)=+\infty$ and $\lim _{c \rightarrow+\infty} F(c)=-\infty, X^{\prime}(c)$ is an increasing function for $\underline{B}<$ $c \leq c^{\star}$ and a decreasing function for $c^{\star}<c<\bar{B}$. Since $X^{\prime}(\underline{B})>0$, if $X^{\prime}(\bar{B}) \geq 0$, then $X^{\prime}(c)>0$ for $\underline{B}<c<\bar{B}$. Thus it is sufficient to show that $X^{\prime}(\bar{B}) \geq 0$. So

$$
\begin{aligned}
X^{\prime}(\bar{B})= & -\gamma n_{1} D_{1} \bar{B}^{-\gamma n_{1}-1}-\gamma n_{2} D_{2} \bar{B}^{-\gamma n_{2}-1}+\frac{1}{K} \\
= & -\frac{n_{1}}{n_{1}-n_{2}}\left[\frac{1}{K}+\gamma n_{2}\left(\frac{1}{K}-\frac{1}{r}\right)\right]\left(\frac{B}{\bar{B}}\right)^{1+\gamma n_{1}} \\
& -\frac{n_{2}}{n_{1}-n_{2}}\left[\frac{1}{K}+\gamma n_{1}\left(\frac{1}{K}-\frac{1}{r}\right)\right]+\frac{1}{K} \\
> & -\frac{n_{1}}{n_{1}-n_{2}}\left[\frac{1}{K}+\gamma n_{2}\left(\frac{1}{K}-\frac{1}{r}\right)\right]-\frac{n_{2}}{n_{1}-n_{2}}\left[\frac{1}{K}+\gamma n_{1}\left(\frac{1}{K}-\frac{1}{r}\right)\right]+\frac{1}{K} \\
= & 0,
\end{aligned}
$$

where the second equality is obtained by inserting $D_{1}$ and $D_{2}$ in (3.26) and (3.27), respectively, into this equation, and the inequality from inequality $-(\underline{B} / \bar{B})^{1+\gamma n_{1}}>-1$.

Lemma 4 shows that the wealth process $X(c)$ is an increasing function of consumption $c$. Therefore, based on Lemmas 3 and 4 , we can see that

$$
\frac{\underline{B}}{r}<\underline{z}<\bar{z}<\frac{\bar{B}}{r}
$$

We derive the optimal policies in the next theorem using FOCs (3.4), (3.5), and (3.18).

Theorem 2 The optimal consumption and portfolio policies $\left(c_{t}^{*}, \pi_{t}^{*}\right)$ are given by

$$
\begin{aligned}
c_{t}^{*} & = \begin{cases}\underline{B} \quad \text { for } \underline{B} / r<X_{t}<\underline{z}, \\
\xi_{t} \text { for } \underline{z} \leq X_{t}<\bar{z}, \\
\bar{B} \quad \text { for } X_{t} \geq \bar{z},\end{cases} \\
\pi_{t}^{*} & = \begin{cases}-\frac{\theta}{\sigma\left(m_{2}-1\right)}\left(x-\frac{B}{r}\right) & \text { for } \underline{B} / r<X_{t}<\underline{z}, \\
\frac{\theta}{\sigma \gamma}\left(-\gamma n_{1} D_{1} \xi_{t}^{-\gamma n_{1}}-\gamma n_{2} D_{2} \xi_{t}^{-\gamma n_{2}}+\frac{1}{K} \xi_{t}\right) & \text { for } \underline{z} \leq X_{t}<\bar{z}, \\
\frac{\theta}{\sigma\left(m_{1}-1\right)}\left(\frac{\bar{B}}{r}-x\right) & \text { for } \bar{z} \leq X_{t}<\bar{B} / r, \\
0 & \text { for } X_{t} \geq \bar{B} / r,\end{cases}
\end{aligned}
$$

where $\xi_{t}\left(\xi_{0}=\xi\right)$ is determined by the algebraic equation as follows:

$$
X_{t}=D_{1} \xi_{t}^{-\gamma n_{1}}+D_{2} \xi_{t}^{-\gamma n_{2}}+\frac{1}{K} \xi_{t}
$$

\section{Numerical implications}

In this section, we take the numerical results about the optimal consumption and portfolio into account. The baseline parameter values from Bensoussan et al. [1] are given as follows:

$$
\beta=0.0371, \quad b=0.1123, \quad r=0.0371, \quad \sigma=0.1954, \quad \gamma=2 \text {. }
$$




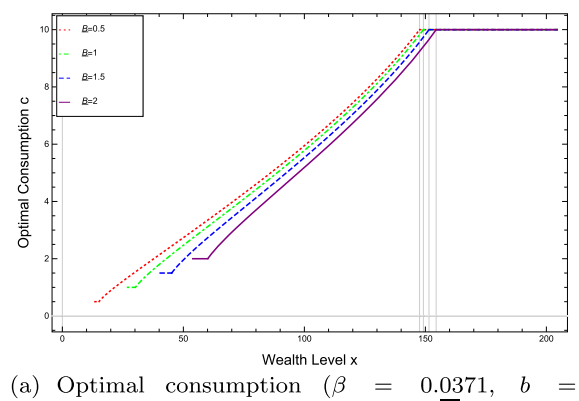

(a) Optimal consumption $(\beta=0.0371, \quad b$
$0.1123, r=0.0371, \sigma=0.1954, \gamma=2, \bar{B}=10)$

Figure 1 The optimal consumption/portfolio when lower bound on consumption $\underline{B}$ increases

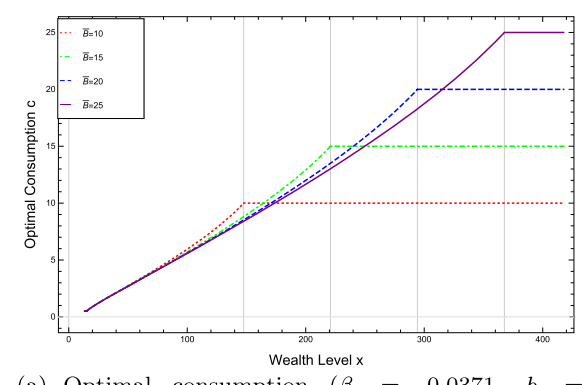

(a) Optimal consumption $(\beta=0.0371, b=$ $0.1123, r=0.0371, \sigma=0.1954, \gamma=2, \underline{B}=0.5)$

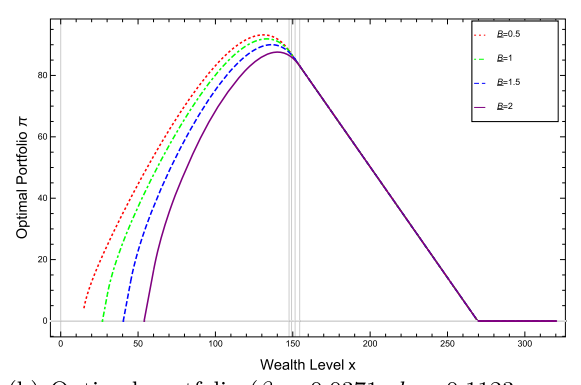

(b) Optimal portfolio $(\beta=0.0371, b=0.1123, r=$ (b) Optimal portfolio $(\beta=0.0371$,

Figure 2 The optimal consumption/portfolio when upper bound on consumption $\bar{B}$ increases

In Figs. 1(a) and 1(b), we fix $\bar{B}=10$. As shown in Fig. 1(a), the optimal consumption becomes lower as the subsistence consumption level rises; that is, as the constraint becomes stronger, the agent's consumption shrinks. Similarly, as shown in Fig. 1(b), the optimal portfolio becomes lower as the subsistence consumption level rises; that is, as the constraint becomes stronger, the agent's investment shrinks. In addition, the critical wealth level $\bar{z}$ becomes higher as the subsistence consumption level rises. Since the investment is lower when the subsistence consumption level is higher, a higher critical wealth level is required for the same upper bound on consumption.

In Figs. 2(a) and 2(b), we fix $\underline{B}=0.5$. As shown in Fig. 2(a), the optimal consumption and critical wealth level $\bar{z}$ become higher as the upper bound on consumption rises. This is because the agent consumes more in order to reach the higher upper bound on consumption and a higher critical wealth level. As shown in Fig. 2(b), the peak of the optimal portfolio becomes higher as the upper bound on consumption rises. This is because the agent invests more in order to reach the critical wealth level $\bar{z}$ quickly when the upper bound on consumption is higher.

From Figs. 1(b) and 2(b), we can observe the decreasing aspect of the optimal portfolio after a certain wealth level. Thus, we can see that the upper bound on consumption provides a bliss level in a quadratic utility model (see $[6,12])$. The decreasing features of the optimal portfolio are remarkable effects of an upper bound on consumption. 


\section{Concluding remarks}

Since we have previously taken the optimal consumption and portfolio choice problem into account with quadratic utility [12], we can observe the aspect of the bliss level of consumption. This motivated us to consider an upper bound on consumption with CRRA utility. By investigating the optimal consumption/investment problem under the consumption constraints, we obtain that the upper bound on consumption acts like a bliss level in a quadratic utility model. Furthermore, we consider two boundaries, lower and upper bounds, on consumption and use the dynamic programming method to derive closedform solutions. We also observe the effects of the optimal consumption and portfolio on consumption constraints and present some numerical implications.

\section{Acknowledgements}

We are indebted to two anonymous referees for valuable advices and useful comments, which have improved our paper essentially.

\section{Funding}

Kum-Hwan Roh was supported by the National Research Foundation of Korea (NRF) grant [Grant No.

NRF-2020R1F1A1A01076001], and Yong Hyun Shin was supported by NRF grant [Grant No. NRF-2020R1A2C1A01006134]

\section{Availability of data and materials}

Data sharing not applicable to this article as no datasets were generated or analyzed during the current study.

\section{Competing interests}

The authors declare that they have no competing interests.

\section{Authors' contributions}

All authors contributed equally to the writing of this paper. All authors read and approved the final manuscript.

\section{Author details}

'Department of Mathematics, Hannam University, Daejeon, 34430, Republic of Korea. ${ }^{2}$ Department of Mathematics \& Research Institute of Natural Sciences, Sookmyung Women's University, Seoul, 04310, Republic of Korea.

\section{Publisher's Note}

Springer Nature remains neutral with regard to jurisdictional claims in published maps and institutional affiliations.

Received: 25 March 2020 Accepted: 29 June 2020 Published online: 08 July 2020

\section{References}

1. Bensoussan, A., Jang, B.-G., Park, S., Unemployment risks and optimal retirement in an incomplete market. Oper. Res. 64, 1015-1032 (2016)

2. Gong, N., Li, T.: Role of index bonds in an optimal dynamic asset allocation model with real subsistence consumption. Appl. Math. Comput. 174, 710-731 (2006)

3. Jang, B.-G., Koo, H.K., Park, S.: Optimal consumption and investment with insurer default risk. Insur. Math. Econ. 88 44-56 (2019)

4. Jian, X., Yi, F., Zhang, J.: Investment and consumption problem in finite time with consumption constraint. ESAIM Control Optim. Calc. Var. 23, 1601-1615 (2017)

5. Karatzas, l., Lehoczky, J.P., Sethi, S.P., Shreve, S.E.: Explicit solution of a general consumption/investment problem. Math. Oper. Res. 11, 261-294 (1986)

6. Koo, J.L., Ahn, S.R., Koo, B.L., Koo, H.K., Shin, Y.H.: Optimal consumption and portfolio selection with quadratic utility and a subsistence consumption constraint. Stoch. Anal. Appl. 34, 165-177 (2016)

7. Lakner, P., Nygren, L.M.: Portfolio optimization with downside constraints. Math. Finance 16, 283-299 (2006)

8. Ma, Q., Yi, F., Guan, C.: A consumption-investment problem with constraints on minimum and maximum consumption rates. J. Comput. Appl. Math. 338, 185-198 (2018)

9. Merton, R.C.: Lifetime portfolio selection under uncertainty: the continuous-time case. Rev. Econ. Stat. 51, 247-257 (1969)

10. Merton, R.C.: Optimum consumption and portfolio rules in a continuous-time model. J. Econ. Theory 3, 373-413 (1971)

11. Shim, G., Shin, Y.H.: An optimal job, consumption/leisure, and investment policy. Oper. Res. Lett. 42, 145-149 (2014)

12. Shin, Y.H., Koo, J.L., Roh, K.-H.: An optimal consumption and investment problem with quadratic utility and subsistence consumption constraints: a dynamic programming approach. Math. Model. Anal. 23, 627-638 (2018)

13. Shin, Y.H., Lim, B.H., Choi, U.J.: Optimal consumption and portfolio selection problem with downside consumption constraints. Appl. Math. Comput. 188, 1801-1811 (2007)

14. Xu, Z.Q., Yi, F.: An optimal consumption-investment model with constraint on consumption. Math. Control Relat. Fields 6, 517-534 (2016)

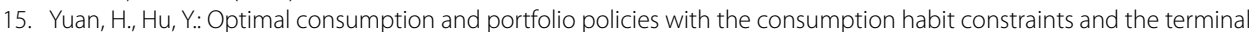
wealth downside constraints. Insur. Math. Econ. 45, 405-409 (2009) 